\title{
Coherent Peaks and Minimal Probing Depth in Photoemission Spectroscopy of Mott-Hubbard Systems
}

\author{
G. Panaccione, ${ }^{1}$ M. Altarelli, ${ }^{2,3}$ A. Fondacaro, ${ }^{4}$ A. Georges,${ }^{5}$ S. Huotari, ${ }^{6}$ P. Lacovig, ${ }^{7}$ A. Lichtenstein, ${ }^{8}$ P. Metcalf, ${ }^{9}$ \\ G. Monaco, ${ }^{6}$ F. Offi, ${ }^{4}$ L. Paolasini, ${ }^{6}$ A. Poteryaev, ${ }^{5}$ O. Tjernberg, ${ }^{10}$ and M. Sacchi ${ }^{10}$ \\ ${ }^{1}$ TASC Laboratory, INFM-CNR, in Area Science Park, S.S.14, Km 163.5, I-34012 Trieste, Italy \\ ${ }^{2}$ Abdus Salam International Centre for Theoretical Physics, Strada Costiera 11, I-34014 Trieste, Italy \\ ${ }^{3}$ European XFEL Project Team clo DESY, Notkestrasse 85, D-22607 Hamburg, Germany \\ ${ }^{4}$ Dipartimento di Fisica, University Roma III, Via della Vasca Navale 84, I-00146 Roma, Italy \\ ${ }^{5}$ Centre de Physique Théorique, Ecole Polytechnique, F-91128 Palaiseau Cedex, France \\ ${ }^{6}$ European Synchrotron Radiation Facility, BP 220, F-38042 Grenoble Cedex 9, France \\ ${ }^{7}$ Sincrotrone Trieste S.C.p.A., S.S. 14 Km 163.5, in Area Science Park, I-34012 Trieste, Italy \\ ${ }^{8}$ Institut für Theoretische Physik, Universität Hamburg, Jungiusstraße 9, D-20355 Hamburg, Germany \\ ${ }^{9}$ Department of Chemistry, Purdue University, West Lafayette, Indiana 47907, USA \\ ${ }^{10}$ LMSP, Royal Institute of Technology, Electrum 229, S-16440 Kista, Sweden \\ ${ }^{11}$ Laboratoire Chimie Physique LCP-MR, Université Pierre et Marie Curie, 75005 Paris, France \\ and Synchrotron SOLEIL, Boîte Postale 48, 91192 Gif-sur-Yvette, France
}

(Received 10 April 2006; published 11 September 2006)

\begin{abstract}
We have measured hard $\mathrm{x}$-ray photoemission spectra of pure vanadium sesquioxide $\left(\mathrm{V}_{2} \mathrm{O}_{3}\right)$ across its metal-insulator transition. We show that, in the metallic phase, a clear correlation exists between the shakedown satellites observed in the vanadium $2 p$ and $3 p$ core-level spectra and the coherent peak measured at the Fermi level. Comparing experimental results and dynamical mean-field theory calculations, we estimate the Hubbard energy $U$ in $\mathrm{V}_{2} \mathrm{O}_{3}(4.20 \pm 0.05 \mathrm{eV})$. From our bulk-sensitive photoemission spectra we infer the existence of a critical probing depth for investigating electronic properties in strongly correlated solids.
\end{abstract}

DOI: 10.1103/PhysRevLett.97.116401

PACS numbers: 71.27.+a, 71.30.+h

The understanding of how electrons behave and interact in a solid is one of the major challenges in solid state physics. Electron correlations play a major role in determining many exotic properties of solid systems, such as superconductivity and colossal magnetoresistance. A remarkable example is the Mott metal-to-insulator transition (MIT) in transition metal oxides (TMO), whose occurrence depends on the competition between itinerancy and correlation of electrons. The Mott-Hubbard theory describes the MIT via a reduction of the parameter $W / U[1,2]$, where $W$ is the bandwidth and the Hubbard energy $U$ is the effective on-site repulsion. However, metals in the strongly correlated regime (i.e., $W / U$ close to the critical ratio associated with the MIT) display a richer excitation spectrum: Hubbard bands (albeit broadened) are observed at high energies [3], while low-energy quasiparticle states are present close to the Fermi energy $E_{F}$. Over the last decade, the dynamical mean-field theory (DMFT) has successfully described in a unified approach both the electron-electron interaction and the density of states effects across the MIT [4,5], the coexistence of Hubbard bands and quasiparticle features being one of its foremost predictions [6]. On the experimental side, photoemission spectroscopy (PES) is the technique best suited to directly reveal this coexistence, but reliable comparisons between DMFT and PES data across the MIT are rare: this is mainly due to the lack of bulk-sensitive PES experiments.
Vanadium sesquioxide, $\mathrm{V}_{2} \mathrm{O}_{3}$ is a paradigmatic example of a Mott-Hubbard system: its phase diagram presents a Mott transition as a function of either temperature, pressure or doping [2]. At ambient temperature and pressure, pure $\mathrm{V}_{2} \mathrm{O}_{3}$ crystallizes in the $\alpha$-corundum structure and undergoes a first order transition at $150 \mathrm{~K}$, passing from the paramagnetic metallic (PM) phase to the antiferromagnetic insulating (AFI) phase, with monoclinic structure. The MIT is destructive in pure $\mathrm{V}_{2} \mathrm{O}_{3}$, due to the large volume increase, and can result in the sample turning to powder. In general, the surface preparation of $\mathrm{V}_{2} \mathrm{O}_{3}$ substantially alters its chemical and structural properties [7]. The lowenergy quasiparticle features of $\mathrm{V}_{2} \mathrm{O}_{3}$ in the $\mathrm{PM}$ phase have been identified recently thanks to the increased bulk sensitivity obtained by PES in the soft $x$-ray regime, but the experimental width and the weight between coherent and incoherent intensity are not well reproduced by the theory [8], and still a large spread exists in the estimated values of the Hubbard $U$ correlation term [9].

Furthermore, the recent use of hard X-ray photoemission (HAXPES) has revealed the existence of sharp satellite structures in core-level spectra of $3 d \mathrm{TMO}$, including the case of $\mathrm{V}_{1.98} \mathrm{Cr}_{0.02} \mathrm{O}_{3}$. These features, known as shakedown satellites or well-screened peaks in $4 f$ and $4 d$ systems $[10,11]$, are practically absent in soft $\mathrm{x}$-ray PES from $3 d$-based TMO, thus suggesting a significant change in the screening mechanism between surface and volume 
[12-15]. The theoretical description of HAXPES data, based on (i) the combination between local and nonlocal screening and (ii) the presence of quasiparticle peaks at $E_{F}$, is still under debate [16,17].

Although bulk sensitivity has been the common parameter to obtain new insight into the physics of $\mathrm{V}_{2} \mathrm{O}_{3}$, and Mott-Hubbard systems in general, the link between corelevel satellites and states at $E_{F}$ has, so far, little experimental support [17]. To address this point, we have performed HAXPES on pure $\mathrm{V}_{2} \mathrm{O}_{3}$ single crystals using a photon energy of $\hbar \omega \approx 5.95 \mathrm{keV}$, able to enhance the depth sensitivity of the measurements up to $\approx 150 \AA$ of information depth [18]. We have modeled, via LDA + DMFT calculations, the transfer of spectral weight observed across the MIT in the valence band region, obtaining a satisfactory agreement with experimental data and a quantitative estimate of the Hubbard $U$ correlation term. We show that a clear relationship exists between the coherent peak measured near $E_{F}$ and the sharp satellites observed in the vanadium $2 p$ core-level spectra, both features pertaining to the metallic phase only. Our results suggest the existence of a minimal probing depth for investigating bulk electronic properties in strongly correlated solids.

Well-annealed single crystals of $\mathrm{V}_{2} \mathrm{O}_{3}$, oriented to have their cleavage plane perpendicular to the (0001) axis, were grown at Purdue University [19] and characterized by x-ray diffraction and SQUID measurements. HAXPES measurements were performed using a dedicated setup [20] installed on the ID16 beam line of the ESRF synchrotron radiation source. The $\mathrm{x}$-ray spot size was $50 \times 120 \mu \mathrm{m}^{2}$ and the overall energy resolution (photons + analyzer) was set to either $250 \mathrm{meV}$, for valence band spectra, or $450 \mathrm{meV}$, for core levels. When not specified otherwise, we present data obtained on samples cleaved in air and immediately inserted in vacuum. Identical results have been obtained consistently on several samples.

In Fig. 1 we present valence band spectra compared to DMFT calculations $[4,21]$. Raw data in panel (a) show a well-defined coherent peak at $E_{F}$ in the PM phase, together with an incoherent band centered at around $1.5 \mathrm{eV}$ binding energy [22]. When lowering the temperature to $140 \mathrm{~K}$ (AFI phase) the coherent peak disappears, its intensity being transferred to a broad band extending from 0.5 to $2.0 \mathrm{eV}$. Moreover, we observe the opening of a gap of $220 \pm$ $20 \mathrm{meV}$. We have carried out the calculations for various values of the interacting parameter $U$, finding that $U=$ $4.2 \mathrm{eV}$ provides the best simultaneous description of the three phases observed in $\mathrm{V}_{2} \mathrm{O}_{3}$, i.e., paramagnetic metal, paramagnetic insulator (not reported here) and antiferromagnetic insulator. The value of the screened Coulomb interaction depends on the choice of local orbitals and a basis set. However, once the latter are fixed, the value of $U$ can be adjusted to insure good agreement with one or more experimental data and the precision of such determination of $U$ can be estimated. In our case we aimed for a con-
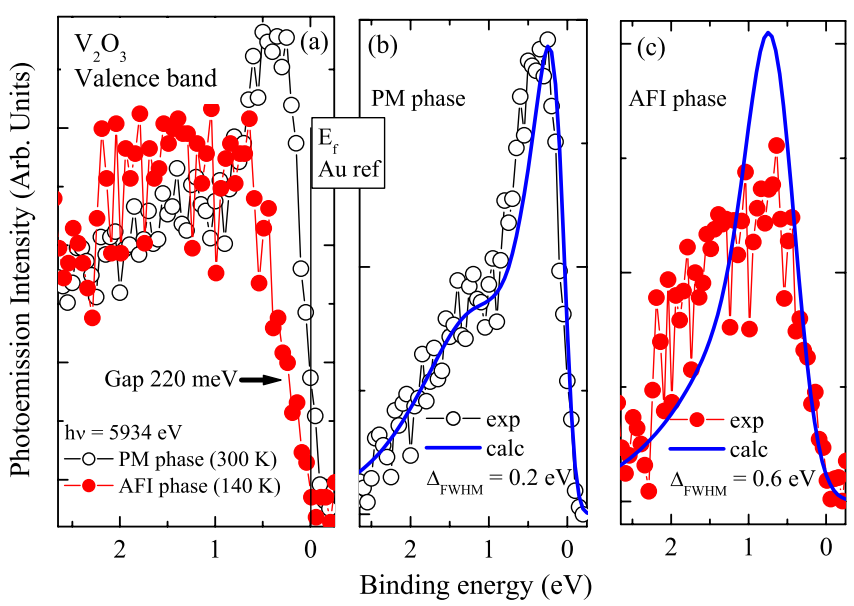

FIG. 1 (color online). Valence band spectra in the PM (white circles) and AFI (filled red circles) phase. (a) Raw data with no background subtraction. After subtraction of an integral background, spectra of panel (a) are compared with DMFT calculations for the PM phase (panel b) and for the AFI phase (panel c). To match the leading edge of the experimental data, calculated line shapes are convoluted with a Gaussian whose FWHM is $\Delta=0.25 \mathrm{eV}$ (PM phase) or $\Delta=0.6 \mathrm{eV}$ (AFI phase).

sistent physical picture of all phases of $\mathrm{V}_{2} \mathrm{O}_{3}$ and concluded that $U=4.20 \pm 0.05 \mathrm{eV}$. The calculated value of the gap obtained in the AFI phase is about $0.65 \mathrm{eV}$, in good agreement with optical measurement [7]. Values of $U$ larger than $4.2 \mathrm{eV}$ result in an increased AFI gap and in the opening of a gap also for the structure corresponding to the PM phase. Panels (b) and (c) show LDA + DMFT calculations obtained with $U=4.2 \mathrm{eV}$ convoluted with a Gaussian broadening (FWHM $=0.25 \mathrm{eV}$ in the PM phase) to account for the finite energy resolution. In the PM phase (panel b) the agreement is remarkably good, the calculation matching simultaneously the ratio between coherent and incoherent parts as well as their location on the energy scale. In the calculations, the change in intensity of the coherent peak in the metallic phase, when temperature is varied from 400 to $1200 \mathrm{~K}$, was found to be less than $10 \%$. This confirms the observed temperature independent behavior of the core-level satellites in Fig. 2(b), when approaching the MIT temperature. In the AFI phase (panel c), calculations give the correct energy position of the intensity maximum and the value of the experimental gap is well reproduced. The intensity at the leading edge close to $E_{F}$ can be matched better by increasing the broadening to $0.6 \mathrm{eV}$, but the agreement remains qualitative at higher binding energies. One notices that the experimental intensity spreads over a larger bandwidth than in DMFT calculations. The larger experimental width may partly justify the disagreement, in terms of intensity, between experimental and calculated AFI spectrum. At present, we have no explanation for such difference.

Panel (a) of Fig. 2 shows the V $2 p$ core-level spectra in the PM and AFI phases. In the PM spectrum, each spinorbit split partner displays a shakedown, or well-screened, 

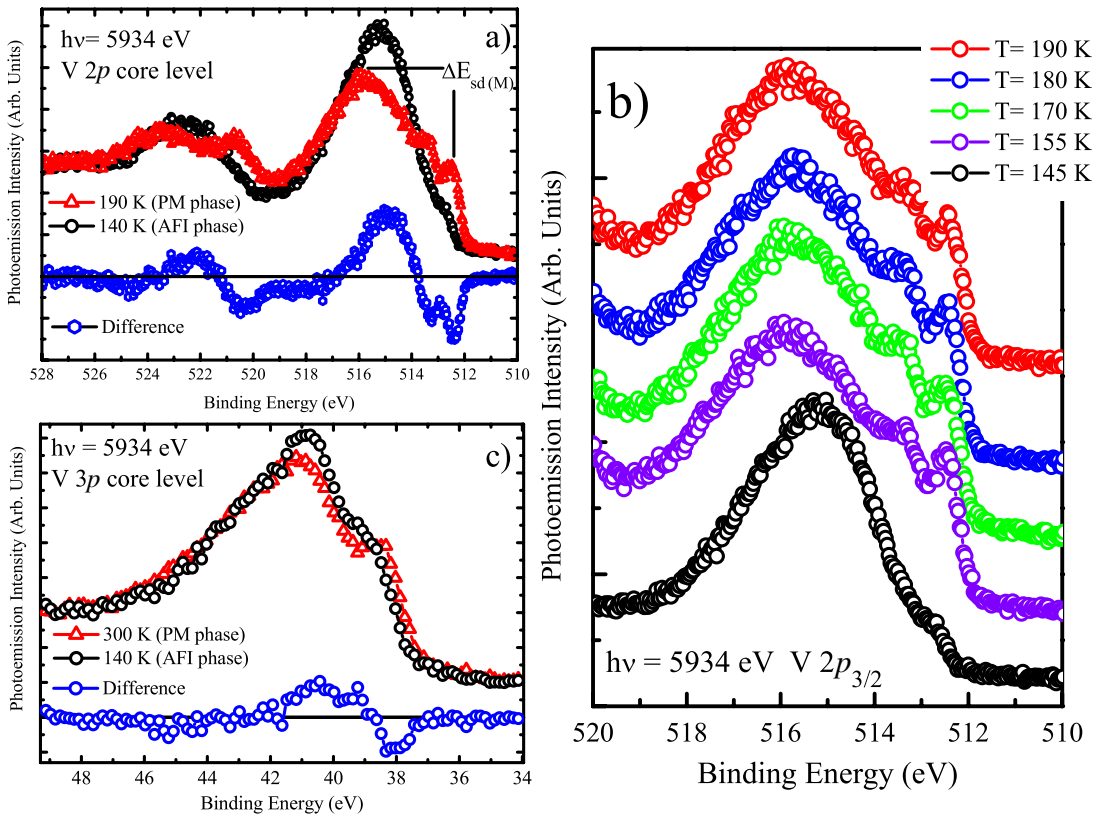

FIG. 2 (color online). HAXPES core-level spectra. Panel (a): V $2 p$ core-level spectra in the PM (red triangles) and the AFI phase (black circles), together with their difference spectrum (blue circles). Spectra have been normalized to match the intensity either side of the peaks. Panel (b): Temperature dependence of the V $2 p_{3 / 2}$ shakedown peak. Spectra are shifted along the intensity axis for better comparison. Panel (c): V $3 p$ core-level spectra in the PM phase (red triangles) and in the AFI phase (black circles), together with their difference (blue circles).

satellite peak, placed at $512.4 \mathrm{eV}\left(2 p_{3 / 2}\right)$ and $520.4 \mathrm{eV}$ $\left(2 p_{1 / 2}\right)$ of binding energy. The energy separation between the satellite and the main peak $\Delta E_{\mathrm{sd}(M)}$ is $3.25 \pm 0.10 \mathrm{eV}$ for the $2 p_{3 / 2}$, and $3.00 \pm 0.20 \mathrm{eV}$ for $2 p_{1 / 2}$. The main features of the spectra agree with previous data obtained on $\mathrm{V}_{1.98} \mathrm{Cr}_{0.02} \mathrm{O}_{3}$ in the HAXPES regime [13]. The clear separation between main peak and satellite, a special feature of HAXPES measurements not observed in conventional surface sensitive XPS [23,24], allows us to estimate the transfer of spectral weight across the MIT. The integrated intensity of the difference curve (blue circles in panel a) averages to zero, within the error bar. In panel b), the $2 p_{3 / 2}$ shakedown feature displays a constant spectral weight vs $T$ and disappears abruptly when the MIT takes place at $150 \mathrm{~K}$, leaving the $2 p$ spectrum almost featureless, with only a small shoulder on the low-energy side of the $2 p_{3 / 2}$ spectrum. A similar transfer of spectral weight is found at the shallower V $3 p$ core-level (panel c) and a satellite peak is clearly visible on the low-energy side, at $38.3 \mathrm{eV}$ binding energy.

Shakedown satellites are caused by a more efficient channel for screening the core hole, expressing the extra (attractive) interaction between the core-hole and $d$ electrons in the final state of the photoionization process [25]. Recently, the presence and the evolution of shakedown satellites in the $4 d$ PES spectra of ruthenates have been explained in terms of a Mott-Hubbard picture within a DMFT approach [17]. In the insulating phase, the (downward) shift in energy of the shakedown satellite with respect to the main line was estimated to be $Q-U$ in the "atomic" limit $W / U \rightarrow 0$. In this expression, $-Q$ $(Q>0)$ is the attractive interaction between the corehole and a $d$ electron. We have generalized the analysis of Ref. [17], where particle-hole symmetry was assumed. This leads to an estimate of the energy shift of the shakedown satellite which reads: $(Q-U)-\varepsilon_{d}=\Delta E_{\mathrm{sd}(M)}$ [see Fig. 2(a)]. In this expression, $\varepsilon_{d}$ is the nominal position of the effective atomic $d$ level counted from the Fermi level $\left(\varepsilon_{d}<0\right)$, which can be taken as the center of gravity of the lower Hubbard band. The states entering the screening process of the core hole now involve the quasiparticle resonance at the Fermi level. This sets a theoretical basis for the common origin of the shakedown satellite and of the quasiparticle resonance at the Fermi level that, as shown below, we experimentally observe in the metallic phase.

Figure 3 compares the intensity of the V $2 p_{3 / 2}$ shakedown satellite and of the corresponding quasiparticle peaks (inset) for two sample preparations, measured at room temperature (PM phase). Sample 1, obtained by cleaving, is characterized by a macroscopically flat surface area. Sample 2, mechanically scraped, displays an increased roughness over the whole surface. Figure 3 shows unambiguously that the presence of the core-level satellites is directly related to the valence band coherent peaks, and that macroscopic imperfections (sample 2) depress both the satellite intensity and the coherent intensity at $E_{F}$. When the coherent peak is present, a transfer of spectral weight occurs at the MIT in both valence band (gap opening) and core-level spectra. Scraped samples do not display any gap opening, and coherent intensity is suppressed both at $E_{F}$ and at the core level. Shakedown intensities have 


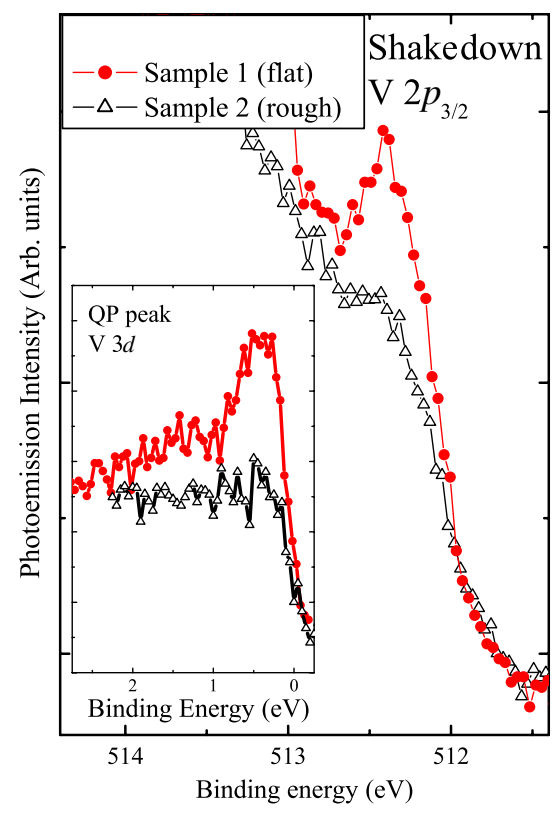

FIG. 3 (color online). Room temperature V $2 p_{3 / 2}$ core level and valence band (inset) for cleaved flat surface (filled circles) and mechanically polished rough surface (open triangles). Spectra have been arbitrarily scaled, by a multiplication factor, for better comparison.

been already reported in the soft x-ray regime for correlated systems, such as ruthenates and cerium compounds $[10,11]$. However, the intense shakedown satellites so clearly observed in HAXPES of $3 d$ TMOs appear at best as weak shoulders in lower energy PES experiments [1215]. The main difference between the two experiments is the probing depth. If one defines the information depth as the thickness from which $90 \%$ of the PES signal originates, HAXPES reaches $150-200 \AA$ at $6 \mathrm{keV}$ kinetic energy, to be compared with 5-10 $\AA$ and $<30 \AA$ of ultraviolet photoemission spectroscopy (UPS) and $\mathrm{x}$-ray photoemission spectroscopy (XPS) regimes, respectively [18]. Moreover, as shown in Fig. 3, the perturbation of the electronic properties due to a macroscopic roughness of the surface propagates so deeply into the solid that even HAXPES results can be affected. The depth sensitivity argument nicely matches recent valence band results obtained on $\mathrm{V}_{2} \mathrm{O}_{3}$ with soft $\mathrm{x}$ rays [8], where a clear coherent peak has been measured. At the same time, authors stress the role of surface inhomogeneities and the importance of using a small photon spot, thus corroborating the hypothesis that sample topography has a strong influence on electron correlation. Therefore, we are dealing with two distinct aspects: surface quality and probing depth. As expected, the larger probing depth at high kinetic energies relaxes some constraints on surface preparation, but, surprisingly, HAXPES spectra look different with respect to both XPS or UPS, revealing clear coherent peaks, no matter how perfect is the surface. Although further investigation is needed, HAXPES results suggest the presence of a minimum distance from the surface, or effective depth, before the truly electronic properties of the sample can be probed by PES.

In summary, thanks to the enhanced bulk sensitivity of HAXPES measurements on $\mathrm{V}_{2} \mathrm{O}_{3}$, we establish a clear relationship between the coherent intensity at $E_{F}$ and the shakedown satellites of the core levels. The experimental ratio between coherent and incoherent intensity in the metallic phase is in good agreement with DMFT calculations, showing that a realistic value of the Hubbard $U$ correlation term in pure $\mathrm{V}_{2} \mathrm{O}_{3}$ is $4.20 \pm 0.05 \mathrm{eV}$. The present observation sets, in the case of $\mathrm{V}_{2} \mathrm{O}_{3}$, the frame for a complete theoretical description and confirms the remarkable change of the screening properties in strongly correlated systems when going from the surface to the volume.

We acknowledge fruitful discussions with V. Anisimov, P. Torelli, M. Grioni, and I. Vobornik. This work was supported by INFM-CNR and by the EU Project No. HPRI-CT-2001-50032. We are grateful to O.K. Andersen and T. Saha-Dasgupta for a collaboration on the DMFT calculation of the valence spectrum.

[1] N. F. Mott, Metal Insulator Transition (Taylor \& Francis, London, 1990).

[2] M. Imada et al., Rev. Mod. Phys. 70, 1039 (1998).

[3] A. Fujimori et al., Phys. Rev. Lett. 69, 1796 (1992).

[4] A. Georges et al., Rev. Mod. Phys. 68, 13 (1996).

[5] G. Kotliar and D. Vollhardt, Phys. Today 57, No. 3, 53 (2004).

[6] A. Georges and G. Kotliar, Phys. Rev. B 45, 6479 (1992).

[7] G. A. Thomas et al., Phys. Rev. Lett. 73, 1529 (1994).

[8] S.-K. Mo et al., Phys. Rev. Lett. 90, 186403 (2003).

[9] S. Di Matteo et al., Phys. Rev. B 65, 054413 (2002).

[10] O. Gunnarsson and K. Schönhammer, Phys. Rev. Lett. 50, 604 (1983).

[11] J. Fuggle et al., Phys. Rev. Lett. 45, 1597 (1980).

[12] M. Taguchi et al., Phys. Rev. Lett. 95, 177002 (2005).

[13] M. Taguchi et al., Phys. Rev. B 71, 155102 (2005).

[14] K. Horiba et al., Phys. Rev. Lett. 93, 236401 (2004).

[15] H. Tanaka et al., Phys. Rev. B 73, 094403 (2006).

[16] M. van Veenendal, www.aps.anl.gov/Experimental_ Facilities_Division/Synchrotron_Related_Theory/index. html (to be published).

[17] H. D. Kim et al., Phys. Rev. Lett. 93, 126404 (2004).

[18] M. Sacchi et al., Phys. Rev. B 71, 155117 (2005).

[19] H. R. Harrison et al., Mater. Res. Bull. 15, 571 (1980).

[20] P. Torelli et al., Rev. Sci. Instrum. 76, 023909 (2005).

[21] O. K. Andersen and T. Saha-Dasgupta, Phys. Rev. B 62, R16219 (2000).

[22] Because of the low photoionization cross section at $\sim 6 \mathrm{keV}$, the spectrum of the PM phase corresponds to $14 \mathrm{~h}$ of acquisition time.

[23] K.E. Smith and V.E. Heinrich, Phys. Rev. B 50, 1382 (1994).

[24] D. S. Toledano et al., Surf. Sci. 449, 19 (2000).

[25] P. A. Cox, Transition Metal Oxides (Clarendon, Oxford, 1992). 